\title{
Methylselenized Glucose: Improvement of the Stability of Glucose-Supported Selenium via the End-Capping Strategy
}

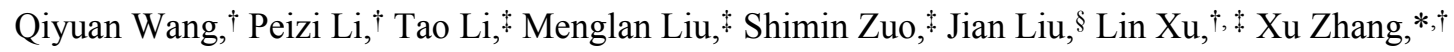
and Lei $\mathrm{Yu}^{*, \dagger}$

$\dagger$ School of Chemistry and Chemical Engineering, Yangzhou University, Yangzhou, Jiangsu 225002, P. R. China

\$ Key Laboratory of Plant Functional Genomics of the Ministry of Education/Jiangsu Key Laboratory of Crop Genomics and Molecular Breeding/Collaborative Innovation of Modern Crops and Food Crops in Jiangsu/Jiangsu Key Laboratory of Crop Genetics and Physiology, College of Agriculture, Yangzhou University, Yangzhou 225009, China

$\S$ Sichuan Selewood Technology Company Limited, Chengdu 610218, China

*Emails: zhangxu@yzu.edu.cn (X. Zhang); yulei@yzu.edu.cn (L. Yu).

\section{CONTENTS}

1. Additional experimental details. S2

2. FT-IR spectra of the materials S4 


\section{Additional experimental details}

(1) Details for XPS sample preparation: The freshly prepared methylselenized glucose (2), as a powder, could be directly sent to XPS analysis (Figure 2a in text). For its aqueous solution that had been exposed in air for 31 days, silica was added into the solution to absorb the materials. Water was then evaporated by rotary evaporator. The obtained powder was dried in vacuum at $80{ }^{\circ} \mathrm{C}$ for $8 \mathrm{~h}$ and then sent to XPS analysis (Figure $2 b$ in text).

(2) Details for planting the Se-enriched rice using the selenized glucose (1) and methylselenized glucose (2): The Se fertilizers 1 or $\mathbf{2}$ containing $220 \mathrm{mg}$ of Se were diluted to $25 \mathrm{~L}$ aqueous solution for $1 \mathrm{mu}\left(666.7 \mathrm{~m}^{2}\right)$ of land. The solution was sprayed on the leaves of the rice at the heading and flower stages for one time.

\section{FT-IR spectra of the materials}

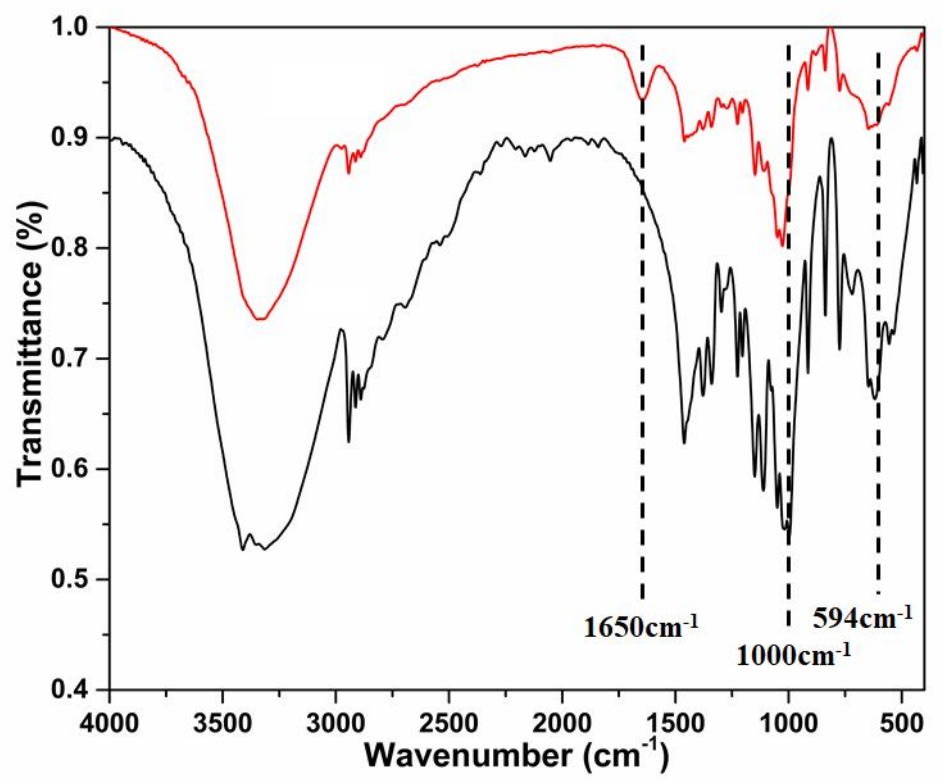

Figure S1. FT-IR spectra of simple glucose (black) and the methylselenized glucose (red). 